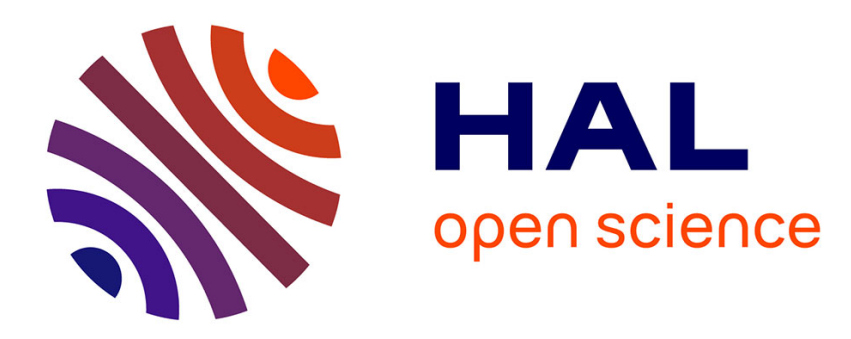

\title{
A Note on Continuous Delayed Sliding Mode Control
}

Denis Efimov, Andrey Polyakov, Leonid Fridman, Wilfrid Perruquetti, Jean-Pierre Richard

\section{To cite this version:}

Denis Efimov, Andrey Polyakov, Leonid Fridman, Wilfrid Perruquetti, Jean-Pierre Richard. A Note on Continuous Delayed Sliding Mode Control. Proc. ECC'15, Jul 2015, Linz, Austria. hal-01140334

\section{HAL Id: hal-01140334 \\ https://hal.inria.fr/hal-01140334}

Submitted on 9 Apr 2015

HAL is a multi-disciplinary open access archive for the deposit and dissemination of scientific research documents, whether they are published or not. The documents may come from teaching and research institutions in France or abroad, or from public or private research centers.
L'archive ouverte pluridisciplinaire HAL, est destinée au dépôt et à la diffusion de documents scientifiques de niveau recherche, publiés ou non, émanant des établissements d'enseignement et de recherche français ou étrangers, des laboratoires publics ou privés. 


\title{
A Note on Continuous Delayed Sliding Mode Control
}

\author{
Efimov D., Polyakov A., Fridman L., Perruquetti W., Richard J.-P.
}

\begin{abstract}
A new continuous sliding mode control approach is introduced with the dedicated mathematical tools. A timedelay modification/approximation of sign function is proposed, and it is shown that by substituting this new "sign" realization in the conventional sliding mode algorithms the main advantages of the sliding mode tools are preserved (like time of convergence and uniformity of stability with respect to matched disturbances), while the chattering is seriously reduced. These results are illustrated and confirmed by numerical simulations for the first order sliding mode control and the super-twisting algorithm.
\end{abstract} bility

Index Terms-Sliding mode, Time delay, Input-to-state sta-

\section{INTRODUCTION}

The sliding mode control and estimation algorithms became very popular nowadays due to their strong advantages: finite time of convergence and compensation of matched disturbances [1], [2], [3]. Despite of that the sliding mode solutions have also several drawbacks mainly originated by the impossibility of a perfect practical realization of sliding motion. Among these shortages it is necessary to mention the chattering phenomenon, which is a high frequency oscillation of control signal when trajectories stay around the sliding surface. The appearance of chattering may physically destroy actuator and/or degrade the performance of transients [4], [5]. There are several approaches for chattering reduction [2]. One of the most popular deals with the high order sliding mode algorithms [6], [7], where the discontinuity, for example

$$
\operatorname{sign}[y(t)]=\left\{\begin{array}{ll}
\frac{y(t)}{|y(t)|}, & y(t) \neq 0 \\
0, & y(t)=0
\end{array},\right.
$$

with $y(t) \in \mathbb{R}$ and $|\cdot|$ is the absolute value, appears not in the control/estimated variable itself but in its derivative of higher

D. Efimov, A. Polyakov, W. Perruquetti and J.-P. Richard are with NonA team @ Inria, Parc Scientifique de la Haute Borne, 40 av. Halley, 59650 Villeneuve d'Ascq, France and CRIStAL (UMR-CNRS 9189), Ecole Centrale de Lille, BP 48, Cité Scientifique, 59651 Villeneuve-d'Ascq, France.

L. Fridman is with Departamento de Ingeniería de Control y Robótica, División de Ingeniería Eléctrica, Facultad de Ingeniería UNAM, Edificio T, Segundo piso Ciudad Universitaria D.F., México.

D. Efimov is with Department of Control Systems and Informatics, Saint Petersburg State University of Information Technologies Mechanics and Optics (ITMO), 49 Kronverkskiy av., 197101 Saint Petersburg, Russia.

This work was supported in part by collaboration program CNRS/CONACyT(209731), CONACyT grant 132125, PAPIIT-UNAM grant IN 113613, by the Government of Russian Federation (Grant 074U01) and the Ministry of Education and Science of Russian Federation (Project 14.Z50.31.0031). order [1], [2]. However, since a discontinuity is present in the system closed loop, it may negatively influence the transients through the corresponding derivative (if this derivative has a physical meaning in the system). That is why frequently for practical realization of sliding mode algorithms different approximations of sign functions are used [8], [9], like for instance

$$
\hat{\operatorname{sign}}[y(t)]=\frac{y(t)}{\epsilon+|y(t)|}
$$

with some sufficiently small $\epsilon>0$, or see also [10] for an interesting general sigmoid approximation. Such a control based on approximated sign functions received the name of continuous sliding mode [11], [12], [13]. The main drawback of existent approximations is that a chattering reduction is achieved by a price of quality loss (a practical convergence in the presence of matched disturbances with an exponential rate [8], [14]). In the present work a development of the continuous sliding mode control is presented that is based on a sign approximation using the time-delay framework, which guarantees the quality preservation (there is no static error in the presence of a matched disturbance and the speed of convergence is faster than exponential).

The dynamical systems subjected by a time-delay, whose models are described by functional differential equations, find their applications in different areas of science and technology [15]. Analysis of delay influence on the system stability is critical for many natural and human-developed systems [16], [17], [18]. The problem of stability investigation in timedelay systems is much more complicated than for ordinary differential equations since design of a Lyapunov-Krasovskii functional or a Lyapunov-Razumikhin function is a complex issue.

Usually appearance of a delay in the system leads to the performance degradation and augmentation of analysis complicacy. However in this work we are going to introduce delay in a proper way into the system, in order to make an approximation of the sign function, proving certain performance in the system under chattering reduction. The obtained approximation is very simple and can be easily implemented in control/estimation systems based on the sliding mode algorithms:

$$
\begin{aligned}
\operatorname{sign}_{\tau}[y(t)] & =\frac{y(t)}{\max \{|y(t)|,|y(t-\tau)|\}} \\
& =\frac{\operatorname{sign}[y(t)]}{\max \{1,|y(t-\tau)| /|y(t)|\}},
\end{aligned}
$$

where $y(t) \in \mathbb{R}$ is a variable whose sign has to be evaluated 
at time instant $t \in \mathbb{R}$ and $\tau>0$ is the delay. Obviously,

$$
\operatorname{sign}_{0}[y(t)]=\operatorname{sign}[y(t)] .
$$

In this work the new continuous delayed sliding mode control framework will be substantiated, it is based on such an approximation and admits the following advantages:

- the control algorithms demonstrate faster than exponential convergence to the origin;

- exact cancellation of matched disturbances is preserved despite of approximation;

- chattering reduction.

The outline of this work is as follows. The preliminary definitions for time-delay systems are given in Section 2. The motivating example of the first order sliding mode control algorithm is considered in Section 3. An extension to high order sliding mode algorithms is presented in Section 4. Several examples are considered for illustration of the obtained results.

\section{PRELIMINARIES}

Consider a functional differential equation of retarded type [17]:

$$
d x(t) / d t=f\left(x_{t}, d(t)\right), t \geq 0,
$$

where $x(t) \in \mathbb{R}^{n}$ and $x_{t} \in C_{[-\tau, 0]}$ is the state function, $x_{t}(s)=x(t+s),-\tau \leq s \leq 0$ (we denote by $C_{[-\tau, 0]}$, $0<\tau<+\infty$ the Banach space of continuous functions $\phi:[-\tau, 0] \rightarrow \mathbb{R}^{n}$ with the uniform norm $\|\phi\|=$ $\sup _{-\tau \leq \varsigma \leq 0}|\phi(\varsigma)|$, where $|\cdot|$ is the standard Euclidean norm) ; $d(t) \in \overline{\mathbb{R}}^{m}$ is an essentially bounded measurable input, i.e. $\|d\|_{\infty}=\sup _{t>0}|d(t)|<+\infty ; f: C_{[-\tau, 0]} \times \mathbb{R}^{m} \rightarrow \mathbb{R}^{n}$ is a locally bounded functional, $f(0,0)=0$. The representation (1) includes pointwise or distributed time-delay systems with either constant or variable time delay $\tau(t) \in[0, \tau]$. We assume that solutions of the system (1) satisfy the initial functional condition $x_{0} \in C_{[-\tau, 0]}$.

\section{A. Discontinuous functional differential equations}

It is known from the theory of functional differential equations [17] that under the above assumptions the system (1) with a locally Lipschitz $f$ has a unique solution $x\left(t, x_{0}, d\right)$ satisfying the initial condition $x_{0}$ for the input $d(t)$, which is defined on some finite time interval $[-\tau, T)$ (we will use the notation $x(t)$ to reference $x\left(t, x_{0}, d\right)$ if the origin of $x_{0}$ and $d$ is clear from the context). If function $f(\phi, d)=f_{0}(\phi(0), \phi, d)$, where $f_{0}: \mathbb{R}^{2 n+m} \rightarrow \mathbb{R}^{n}$, and it is discontinuous with respect to $\phi(0)$ and on a zero measure set $\mathcal{N} \subset \mathbb{R}^{n}$ only, following [19], [20] we will consider its multivalued extension (define $B_{\varepsilon}(x)=\left\{y \in \mathbb{R}^{n}:|x-y| \leq \varepsilon\right\}$ as a closed ball of radius $\varepsilon>0$ around $\left.x \in \mathbb{R}^{n}\right)$ :

$$
F(\phi, d)=\bigcap_{\varepsilon>0} \overline{\operatorname{conv}}\left[f_{0}\left\{B_{\varepsilon}(\phi(0)) \backslash \mathcal{N}, \phi, d\right\}\right],
$$

which is non-empty, compact, convex and upper semicontinuous [20] for any $d \in \mathbb{R}^{m}$. In particular, the multivalued extension of $\operatorname{sign}_{\tau}[y(t)]$ can be defined as follows:

$$
\overline{\operatorname{sign}}_{\tau}[y(t)]=\left\{\begin{array}{ll}
\frac{y(t)}{\max \{|y(t)|,|y(t-\tau)|\}}, & y(t) \neq 0 \\
{[-1,1],} & y(t)=0
\end{array} .\right.
$$

In this case, instead of (1), the solutions of the following functional differential inclusion will be considered:

$$
d x(t) / d t \in F\left(x_{t}, d(t)\right), t \geq 0,
$$

and for any initial condition $x_{0} \in C_{[-\tau, 0]}$ the set of corresponding solutions of (2) initiated at $x_{0}$ for the input $d$ can be denoted as $\mathcal{S}\left(x_{0}, d\right)$.

For a locally Lipschitz continuous function $V: \mathbb{R}^{n} \rightarrow \mathbb{R}_{+}$ the upper directional Dini derivative for (2) is defined as follows:

$$
\begin{gathered}
D^{+} V\left[x_{t}(0)\right] F\left(x_{t}, d\right)= \\
\sup _{v \in F\left(x_{t}, d\right)} \lim _{h \rightarrow 0^{+}} \sup \frac{V\left[x_{t}(0)+h v\right]-V\left[x_{t}(0)\right]}{h} .
\end{gathered}
$$

A continuous function $\sigma: \mathbb{R}_{+} \rightarrow \mathbb{R}_{+}$belongs to class $\mathcal{K}$ if it is strictly increasing and $\sigma(0)=0$; it belongs to class $\mathcal{K}_{\infty}$ if it is also radially unbounded. A continuous function $\beta: \mathbb{R}_{+} \times \mathbb{R}_{+} \rightarrow \mathbb{R}_{+}$belongs to class $\mathcal{K} \mathcal{L}$ if $\beta(\cdot, r) \in \mathcal{K}$ and $\beta(r, \cdot)$ is a strictly decreasing to zero for any fixed $r \in \mathbb{R}_{+}$.

\section{B. Stability definitions}

Let $\Omega$ be an open neighborhood of the origin in $C_{[-\tau, 0]}$, and denote $\mathcal{D}$ set of all essentially bounded measurable input $d: \mathbb{R}_{+} \rightarrow \mathbb{R}^{m}$ with $\|d\|_{\infty}<D$ for some $0<D<+\infty$.

Definition 1. [21] The system (2) for $d=0$ is said to be

(a) stable if there is $\sigma \in \mathcal{K}$ such that for any $x_{0} \in \Omega$ and all $x\left(t, x_{0}, 0\right) \in \mathcal{S}\left(x_{0}, 0\right),\left|x\left(t, x_{0}, 0\right)\right| \leq \sigma\left(\left\|x_{0}\right\|\right)$ for all $t \geq 0$

(b) asymptotically stable if it is stable and $\lim _{t \rightarrow+\infty}\left|x\left(t, x_{0}, 0\right)\right|=0$ for any $x_{0} \in \Omega$ and all $x\left(t, x_{0}, 0\right) \in \mathcal{S}\left(x_{0}, 0\right)$;

(c) finite-time stable if it is stable and for any $x_{0} \in \Omega$ there exists $0 \leq T^{x_{0}}<+\infty$ such that $x\left(t, x_{0}, 0\right)=0$ for all $t \geq T^{x_{0}}$ and all $x\left(t, x_{0}, 0\right) \in \mathcal{S}\left(x_{0}, 0\right)$. The functional $T_{0}\left(x_{0}\right)=\inf \left\{T^{x_{0}} \geq 0: x\left(t, x_{0}, 0\right)=0 \forall t \geq T^{x_{0}}\right\}$ is called the settling time of the system (1).

If $\Omega=C_{[-\tau, 0]}$, then the corresponding properties are called global stability/asymptotic stability/finite-time stability.

If the above properties hold for all $x\left(t, x_{0}, d\right) \in \mathcal{S}\left(x_{0}, d\right)$ with any $x_{0} \in \Omega$ and any $d \in \mathcal{D}$, then the corresponding properties are called uniform stability/asymptotic stability/finite-time stability.

For the forthcoming analysis we will need LyapunovRazumikhin theorem, which is given below (the variants for ordinary differential equations are given in [17], [16]).

Theorem 1. Let $\alpha_{1}, \alpha_{2} \in \mathcal{K}$ and $\eta: \mathbb{R}_{+} \rightarrow \mathbb{R}_{+}$be a continuous nondecreasing function. If there exists a Lipschitz continuous function $V: \mathbb{R}^{n} \rightarrow \mathbb{R}$ such that

$$
\alpha_{1}(|x|) \leq V(x) \leq \alpha_{2}(|x|), \forall x \in \mathbb{R}^{n},
$$

and the derivative of $V$ along a solution $x(t)$ of (2) satisfies

$$
D^{+} V[x(t)] F\left(x_{t}, 0\right) \leq-\eta(|x(t)|) \text { if } V[x(t+\theta)] \leq V[x(t)]
$$


for all $\theta \in[-\tau, 0]$, then (2) is stable.

If, in addition, $\eta \in \mathcal{K}$ and there exists a continuous nondecreasing function $p(s)>s$ for $s>0$ such that the condition (3) is strengthened to

$D^{+} V[x(t)] F\left(x_{t}, 0\right) \leq-\eta(|x(t)|)$ if $V[x(t+\theta)] \leq p\{V[x(t)]\}$

for all $\theta \in[-\tau, 0]$, then (2) is asymptotically stable.

If in addition $\alpha_{1} \in \mathcal{K}_{\infty}$, then (2) is globally asymptotically stable. If the above properties are satisfied not only for $d=0$ but for any $d \in \mathcal{D}$, then (2) is uniformly stable/asymptotically stable.

Since the conditions of Theorem 1 are formulated for a Lyapunov function $V$, then the proof is the same for a differential equation or inclusion (it is omitted in this note).

Similarly, in order to analyze robustness of functional differential inclusions we will use an extension of the inputto-state stability (ISS) framework proposed in [22].

Definition 2. The system (2) is called ISS, if there exist $\beta \in \mathcal{K} \mathcal{L}$ and $\gamma \in \mathcal{K}$ such that for all $t \geq 0$ it holds

$$
\left|x\left(t, x_{0}, d\right)\right| \leq \beta\left(\left\|x_{0}\right\|, t\right)+\gamma\left(\|d\|_{\infty}\right)
$$

for all $x_{0} \in C_{[-\tau, 0]}$, all essentially bounded measurable inputs $d$ and all $x\left(t, x_{0}, d\right) \in \mathcal{S}\left(x_{0}, d\right)$.

Definition 3. A locally Lipschitz function $V: \mathbb{R}^{n} \rightarrow \mathbb{R}_{+}$ is called ISS Lyapunov-Razumikhin function for (2), if there exist $\alpha_{1}, \alpha_{2} \in \mathcal{K}_{\infty}$ and $\chi_{\tau}, \chi_{d}, \alpha \in \mathcal{K}$ such that the following conditions hold:

$$
\begin{gathered}
\alpha_{1}(|x|) \leq V(x) \leq \alpha_{2}(|x|) \quad \forall x \in \mathbb{R}^{n} ; \\
V(x(t)) \geq \chi_{\tau}\left[\sup _{\theta \in[-\tau, 0]} V\{x(t+\theta)\}\right]+\chi_{d}(|d(t)|) \\
\Rightarrow D^{+} V[x(t)] F\left(x_{t}, d\right) \leq-\alpha(V[x(t)]),
\end{gathered}
$$

for all solutions of (2) $x\left(t, x_{0}, d\right) \in \mathcal{S}\left(x_{0}, d\right)$ for all $x_{0} \in$ $C_{[-\tau, 0]}$ and all essentially bounded measurable inputs $d$.

Theorem 2. [22] If there exists an ISS Lyapunov-Razumikhin function $V$ for (2) and $\chi_{\tau}(s)<s$ for all $s>0$, then the functional differential inclusion (2) is ISS from $d$ to $x$ with the gain $\gamma(s)=\alpha_{1}^{-1} \circ \chi_{d}(s)$.

\section{APPROXIMATION OF THE FIRST ORDER SLIDING MODE ALGORITHM}

\section{A. The rate of convergence}

First of all, let us consider the system

$$
\dot{x}(t)=-k \operatorname{sign}_{\tau}[x(t)]
$$

for $x_{0} \in C_{[-\tau, 0]}$ with $\tau>0$ and $k>0$, in order to evaluate the rate of convergence in a system with the introduced approximation of the sign function, in this case $F\left(x_{t}, 0\right)=-k \overline{\operatorname{sign}}_{\tau}[x(t)]$. First of all note that for (4) the origin is the equilibrium: once $x\left(t^{\prime}\right)=0$ for some $t^{\prime} \geq 0$, then $x(t)=0$ for all $t \geq t^{\prime}$. For any $x_{0} \in C_{[-\tau, 0]}$ consider

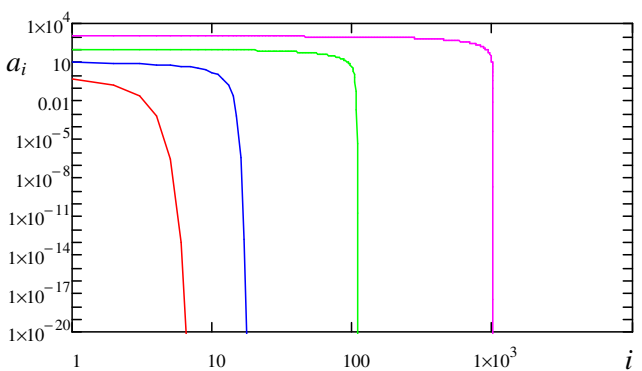

Figure 1. The results of the sequence (5) calculation

a Lyapunov function candidate $V[x(t)]=\frac{1}{2} x^{2}(t)$, for (4) it has the derivative

$$
\begin{gathered}
D^{+} V[x(t)] F\left(x_{t}, 0\right)=-k \frac{x^{2}(t)}{\max \{|x(t)|,|x(t-\tau)|\}} \\
=-k \alpha(t)|x(t)| \leq 0, \alpha(t)=\left\{\begin{array}{ll}
1, & |x(t)| \geq|x(t-\tau)| \\
\frac{|x(t)|}{|x(t-\tau)|}, & |x(t)|<|x(t-\tau)|
\end{array} .\right.
\end{gathered}
$$

Therefore, $V(t)=V[x(t)]$ is not increasing and the system is stable. Using this fact and introducing the sequence of $a_{i}=|x(i \tau)|$ for $i \geq 1$, it is possible to show that

$$
a_{i+1} \leq \frac{a_{i}^{2}}{a_{i}+k \tau}, i \geq 1
$$

Moreover, the rate of convergence of this sequence is polynomial, and for small amplitudes of $x(t)$, the state of (4) converges to zero with a hyper-exponential speed ${ }^{1}$. To confirm this conclusion, the results of this sequence calculation for different initial values of $a_{1}$ and for $k \tau=1$ are shown in Fig. 1 in logarithmic scale.

The following result can be proven (all proofs are excluded due to space limitations).

Lemma 1. The system (4) is globally asymptotically stable, and it has a hyper-exponential rate of convergence.

Consequently, the proven rate of convergence in (4) is not finite-time, and by this it is slower than in the original system

$$
\dot{x}(t)=-k \operatorname{sign}[x(t)],
$$

but anyway it is much faster than an exponential rate, which can be obtained using standard approximations. Another consequence of this result is that in (4) there is no chattering of the right-hand side (that explains the paper title). For an illustration, the results of simulation of (4) and (6) with the discretization step $h=10^{-4}$ are given in Fig. 2 for $x(s)=1$, $s \in[-\tau, 0]$ and $k=\tau=1$. The solid red lines represent the variables $x(t)$ and $\dot{x}(t)$ generated by the system (4), the dash blue lines correspond to the variables of the system (6).

\section{B. Uniform and robust stability/convergence}

Now let us consider the perturbed version of (4):

$$
\dot{x}(t)=-k \operatorname{sign}_{\tau}\left[x(t)+d_{1}(t)\right]+d_{2}(t),
$$

\footnotetext{
${ }^{1}$ I.e. the rate of convergence to the origin is much faster than exponential [23]
} 

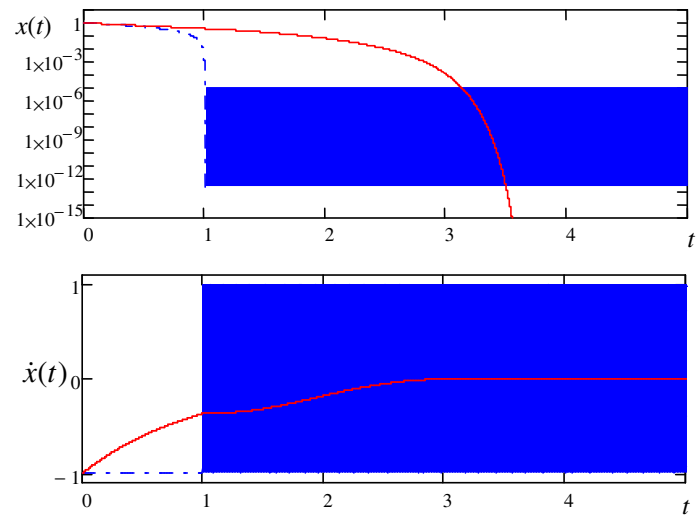

Figure 2. Results of simulation for (4) and (6)

where $d_{1}(t) \in \mathbb{R}$ and $d_{2}(t) \in \mathbb{R}$ represent the measurement noise and the additive disturbance respectively. As usual in the sliding mode theory, we will be interested in the case when $\left\|d_{2}\right\|_{\infty}<D$ and $k>D$, in this case

$$
\begin{aligned}
\dot{x}(t) & \in F\left(x_{t}, d_{1}\right), \\
F\left(x_{t}, d_{1}\right) & =-k \dot{\operatorname{sign}}_{\tau}\left[x(t)+d_{1}(t)\right]+[-D, D] .
\end{aligned}
$$

Consider for (8) a Lyapunov function candidate $V[x(t)]=$ $\frac{1}{2} x^{2}(t)$ :

$D^{+} V[x(t)] F\left(x_{t}, d_{1}\right) \leq-k x(t) \overline{\operatorname{sign}}_{\tau}\left[x(t)+d_{1}(t)\right]+|x(t)| D$.

Let $\frac{\sqrt{2 b}-1}{\sqrt{2 b} p^{-1}+1} k \geq D+\epsilon$ for some $\epsilon>0$, where $0<p<1$ and $b>0.5$, and the following restriction is satisfied on trajectories of (8):

$$
\begin{aligned}
V[x(t)] & \geq b\left|d_{1}(t)\right|^{2}+b\left|d_{1}(t-\tau)\right|^{2}+p^{2} V[x(t-\tau)] \text { (9) } \\
& \geq \max \left\{b\left|d_{1}(t)\right|^{2}, b\left|d_{1}(t-\tau)\right|^{2}, p^{2} V[x(t-\tau)]\right\},
\end{aligned}
$$

then the following relation can be proven under (9):

$$
D^{+} V[x(t)] F\left(x_{t}, d_{1}\right) \leq-\epsilon \sqrt{2 V[x(t)]} .
$$

Thus $V$ is an ISS Lyapunov-Razumikhin function for (8) with respect to an extended input $\left[d_{1}(t) d_{1}(t-\tau)\right]^{T}$ and according to Theorem 2 the functional differential inclusion (8) is ISS from $\left[d_{1}(t) d_{1}(t-\tau)\right]^{T}$ to $x(t)$ :

Lemma 2. Let $\frac{\sqrt{2 b}-1}{\sqrt{2 b} p^{-1}+1} k \geq D+\epsilon$ for some $\epsilon>0$, $0<$ $p<1$ and $b>0.5$. Then (8) is ISS from $d_{1}(t)$ to $x(t)$ with the gain $\gamma(s)=2 \sqrt{2 b} s$.

The results of simulation with the discretization step $h=$ $10^{-4}$ in the Euler method for (7) and the conventional sliding mode system

$$
\dot{x}(t)=-k \operatorname{sign}\left[x(t)+d_{1}(t)\right]+d_{2}(t)
$$

are shown in figures 3 and 4 for $k=1$ and $\tau=1$ (in this case $b=10^{3}, p=0.96$ and $\epsilon=10^{-2}$ for the given $D=0.9$ and $k=1)$. In the top of Fig. 3 the variable $x(t)$ is shown for both (7) (red line) and (10) (blue line), for $d_{1}(t)=0$ and $d_{2}(t)=0.9 \sin (5 t)$. At the bottom of the figure the variable $u(t)=\operatorname{sign}_{\tau}\left[x(t)+d_{1}(t)\right]$ for (7) (red line) and the signal $-d_{2}(t)$ (green dash line) are plotted (the variable

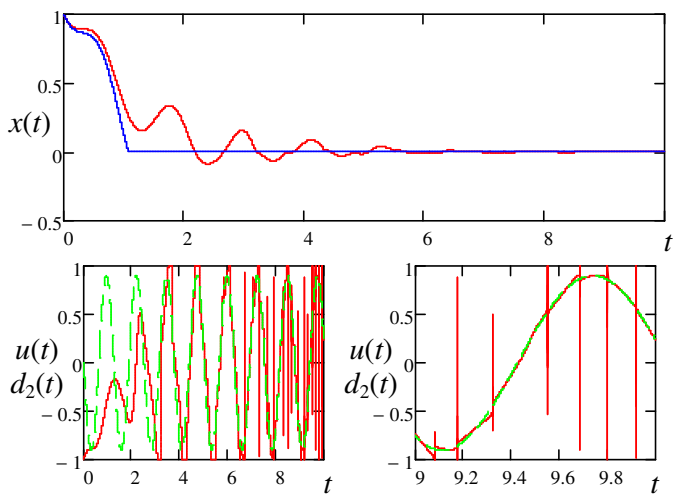

Figure 3. Trajectories of (7) and (10) for $d_{1}=0$

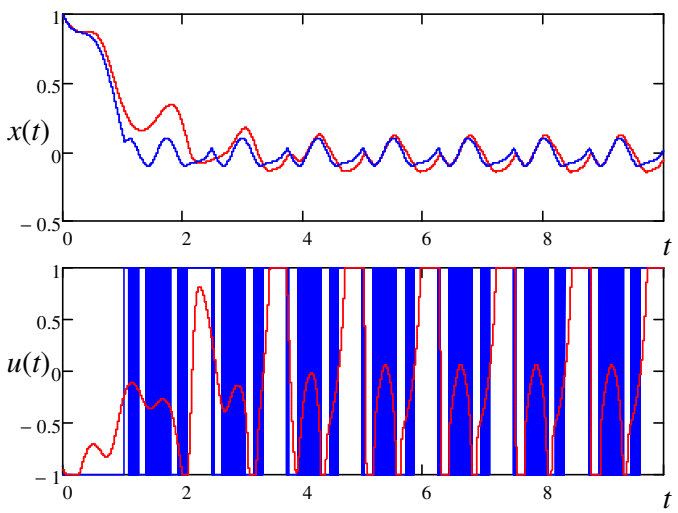

Figure 4. Trajectories of (7) and (10) with $d_{1}(t)=0.1 \sin (10 t)$

$u(t)$ represents a kind of "control" approximation in a sliding mode control system) with its zoomed version for the last second of simulation. The variable $u(t)=\operatorname{sign}\left[x(t)+d_{1}(t)\right]$ for (10) is not shown since it represents a pure chattering after approximately 1 second of time. As we can conclude, the system (7) has much less chattering and its signal $u(t)$ estimates $d_{2}(t)$ directly without a need of filtering, which is usual for conventional sliding mode control and estimation systems. In Fig. 4 the results of simulation are shown for $d_{1}(t)=0.1 \sin (10 t)$ and $d_{2}(t)=0.9 \sin (5 t)$, the variables $x(t)$ and $u(t)$ are shown for (7) (red lines) and (10) (blue lines). In this case, again, the variable $x(t)$ has a similar behavior for (7) and (10), but $u(t)$ contains less chattering for (7).

\section{APPROXIMATION OF HIGH ORDER SLIDING MODE ALGORITHMS}

The main idea of the proof of Lemma 2 has the following interpretation: if for $\operatorname{sign}_{0}\left[x(t)+d_{1}(t)\right]$ the sliding mode system has an ISS Lyapunov function $V$, and since $\operatorname{sign}_{\tau}\left[x(t)+d_{1}(t)\right]$ can be reduced to $\operatorname{sign}_{0}\left[x(t)+d_{1}(t)\right]$ under the Lyapunov-Razumikhin arguments, then $V$ is an ISS Lyapunov-Razumikhin function for the system with $\operatorname{sign}_{\tau}\left[x(t)+d_{1}(t)\right]$. Thus a simple extension for high order sliding mode systems can be obtained. 
Consider a conventional sliding mode system:

$$
\dot{x}(t) \in G(x(t), \overline{\operatorname{sign}}\{s[x(t)]\}, d(t)),
$$

where $x(t) \in \mathbb{R}^{n}$ is the state, $d(t) \in \mathbb{R}^{m}$ is the disturbance, $G: \mathbb{R}^{n+m+k} \rightarrow \mathbb{R}^{n}$ and $s: \mathbb{R}^{n} \rightarrow \mathbb{R}^{k}$ are continuous functions, $s(t)=s[x(t)] \in \mathbb{R}^{k}$ is the sliding surface variable (application of the sign function to a vector argument is understood elementwise).

Assumption 1. Let the system (11) be ISS from $d$ to $x$ and there exist an ISS Lyapunov function $V: \mathbb{R}^{n} \rightarrow \mathbb{R}^{n}$ such that

$$
\begin{gathered}
\alpha_{1}(|x|) \leq V(x) \leq \alpha_{2}(|x|) \quad \forall x \in \mathbb{R}^{n} \\
V(x(t)) \geq \chi_{d}(|d(t)|) \Rightarrow \\
D^{+} V[x(t)] G(x(t), \overline{\operatorname{sign}}\{s[x(t)]\}, d) \leq-\alpha(V[x(t)])
\end{gathered}
$$

for some $\alpha_{1}, \alpha_{2} \in \mathcal{K}_{\infty}$ and $\chi_{d}, \alpha \in \mathcal{K}$.

Let us introduce another time-delayed modification of the sign function for the high order case:

$$
\overline{\operatorname{sign}}_{\tau}[x(t)]= \begin{cases}\frac{s(t)}{\max \{|s(t)|,|s(t-\tau)|\}}, & V[x(t)]<p V[x(t-\tau)] \\ & \text { and }|s(t)| \neq 0 \\ \frac{s(t)}{|s(t)|}, & V[x(t)] \geq p V[x(t-\tau)] \\ & \text { and }|s(t)| \neq 0 \\ {[-1,1]^{k},} & |s(t)|=0\end{cases}
$$

for some constant $0<p<1$, where $V$ has been introduced in Assumption 1. As before, $\overline{\operatorname{sign}}_{0}[x(t)]=\overline{\operatorname{sign}}[x(t)]$.

Theorem 3. Let Assumption 1 be satisfied for the system (11). Then for any $0<\tau<+\infty$ the system

$$
\dot{x}(t) \in G\left(x(t), \overline{\operatorname{sign}}_{\tau}[x(t)], d(t)\right),
$$

is also ISS from $d$ to $x$.

For an illustration of the result of this theorem consider the super-twisting differentiator:

$$
\begin{aligned}
& \dot{x}_{1}(t)=-k_{1} \sqrt{\left|x_{1}(t)\right|} \operatorname{sign}\left[x_{1}(t)\right]+x_{2}(t), \\
& \dot{x}_{2}(t)=-k_{2} \operatorname{sign}\left[x_{1}(t)\right]+d(t),
\end{aligned}
$$

where $0<k_{1}<k_{2}$ are parameters,

$$
G(x, s, d)=\left[\begin{array}{c}
-k_{1} \sqrt{\left|x_{1}\right|} \operatorname{sign}\left[x_{1}\right]+x_{2} \\
-k_{2} s+d
\end{array}\right] .
$$

As a Lyapunov function candidate take one from [24] (the Lyapunov function proposed in [25] also can be used):

$$
V(x)=\xi^{T} P \xi,
$$

where $\xi=\left[\sqrt{\left|x_{1}(t)\right|} \operatorname{sign}\left[x_{1}(t)\right] x_{2}\right]^{T}$ and the matrix $P$ is a solution of the Lyapunov equation $A^{T} P+P A=I$, where $I$ is the identity matrix and

$$
A=\left[\begin{array}{cc}
-0.5 k_{1} & 0.5 \\
-k_{2} & 0
\end{array}\right]
$$

The results of simulation for $k_{1}=1, k_{2}=2, \tau=1$ and $d(t)=\sin (5 t)$ with the discretization step $h=10^{-4}$ in the Euler method are shown in Fig. 5. The red solid lines represent the variables of time-delayed differentiator,

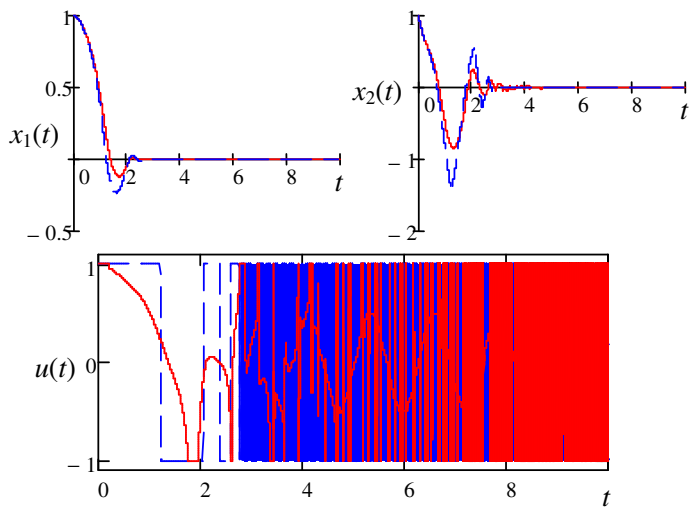

Figure 5. Results of simulation for super-twisting algorithm

the blue dash lines correspond to the variables of (13), the variable $u(t)$ in both cases demonstrate the function $\operatorname{sign}(\cdot)$ behavior and its approximation. As we can conclude from this numerical experiment, the higher order sliding mode algorithm behavior is quite nice and smooth (see the variables $x_{1}(t)$ and $x_{2}(t)$ ), but even in this case a chattering reduction is achieved by a slight decrease of the convergence rate using the new continuous delayed sliding mode algorithm.

\section{CONCLUSions}

In this note the Continuous Delayed Sliding Mode control has been presented, which demonstrates how by a delay introduction in the closed-loop of a nonlinear system it is possible to improve the quality of transients. An approximation of the sign function has been given based on delayed values of the processed variable, which ensures chattering reduction for the sliding mode control and estimation algorithms. The delay has been introduced in a way that under the LyapunovRazumikhin conditions the system is reduced to its original non-delayed form, then its stability can be easily concluded. Efficiency of the proposed approach has been demonstrated on examples: the chattering can be avoided (see Fig. 2) or tolerated (see figures 3-5) by a slight decreasing of the convergence rate, in addition, the matched disturbances are compensated.

\section{REFERENCES}

[1] W. Perruquetti and J. P. Barbot, Sliding Mode Control in Engineering. Marcel Dekker Hardcover, 2002.

[2] L. Fridman, Sliding Mode Enforcement after 1990: Main Results and Some Open Problems, vol. 412 of LNCIS, pp. 3-57. Springer - Verlag Berlin Heidelberg, 2011.

[3] I. Boiko, Discontinuous Control Systems: Frequency-Domain Analysis and Design. Birkhauser, 2009.

[4] V. I. Utkin, Sliding Modes in Control Optimization. Berlin: SpringerVerlag, 1992.

[5] A. Levant, "Chattering analysis," IEEE Transactions on Automatic Control, vol. 55, no. 6, pp. 1380-1389, 2010.

[6] A. Levant, "Sliding order and sliding accuracy in sliding mode control," International Journal of Control, vol. 58, no. 6, pp. 12471263, 1993.

[7] C. Edwards and Y. Shtessel, "Continuous higher order sliding mode control based on adaptive disturbance compensation," in 13th International Workshop on Variable Structure Systems (VSS), pp. 1-5, June 2014. 
[8] G. Ambrosino, G. Celentano, and F. Garofalo, "Robust model tracking control for a class of nonlinear plant," IEEE Trans. on Automatic Control, vol. AC-30, no. 3, pp. 275-279, 1985.

[9] J. Burton and A. Zinober, "Continuous approximation of variable structure control," International Journal of Systems Science, vol. 17, no. 6 , pp. 875-885, 1986.

[10] C. Canudas-de Wit and W. Perruquetti, "Smoothing strategies for highgain control," in Proc. IFAC Latin-American Conference on Automatic Control, (Mexico), 2002.

[11] H. Khalil, Nonlinear Systems. Upper Saddle River, New Jersey: Prentice Hall, 3rd ed., 2002.

[12] H. Oza, Y. Orlov, S. Spurgeon, Y. Aoustin, and C. Chevallereau, "Continuous second order sliding mode based robust finite time tracking of a fully actuated biped robot," in Proc. European Control Conference (ECC), pp. 2600-2605, June 2014.

[13] Y. B. Shtessel, I. A. Shkolnikov, and M. D. Brown, "An asymptotic second-order smooth sliding mode control," Asian Journal of Control, vol. 5, pp. 498-504, 2003.

[14] F. Esfandiari and H. Khalil, "Stability analysis of a continuous implementation of variable structure control," IEEE Trans. on Automatic Control, vol. 36, no. 5, pp. 616-620, 1991.

[15] J. Chiasson and J. Loiseau, eds., Applications of Time Delay Systems, vol. 352 of Lecture Notes in Control and Information Sciences. Springer, 2007.

[16] K. Gu, K. Kharitonov, and J. Chen, Stability of Time-Delay Systems. Control Engineering, Boston: Birkhäuser, 2003.

[17] V. Kolmanovsky and V. Nosov, Stability of functional differential equations. San Diego: CA: Academic, 1986.

[18] J.-P. Richard, "Time-delay systems: an overview of some recent advances and open problems," Automatica, vol. 39, pp. 1667-1694, 2003.

[19] A. F. Filipov, Differential Equations with Discontinuous Righthand Sides. Kluwer Academic Publishers, 1988.

[20] W. Heemels and S. Weiland, "Input-to-state stability and interconnections of dicontinuous dynamical systems," Automatica, vol. 44, no. 12, pp. 3079-3086, 2008

[21] E. Moulay, M. Dambrine, N. Yeganefar, and W. Perruquetti, "Finitetime stability and stabilization of time-delay systems," Systems Control Lett., vol. 57, pp. 561-566, 2008.

[22] A. R. Teel, "Connections between Razumikhin-type theorems and the ISS nonlinear small gain theorem," IEEE Trans. Automat. Control, vol. 43, no. 7, pp. 960-964, 1998.

[23] A. Polyakov, D. Efimov, W. Perruquetti, and J.-P. Richard, "Implicit lyapunov-krasovski functionals for time delay systems," in Proc. 53rd IEEE CDC, (LA), 2014

[24] J. Moreno and A. Osorio, "Strict lyapunov functions for the supertwisting algorithm," IEEE Transactions on Automatic Control, vol. 57 , no. 4, pp. 1035-1040, 2012.

[25] A. Polyakov and A. Poznyak, "Reaching time estimation for "supertwisting" second order sliding mode controller via lyapunov function designing," IEEE Transactions on Automatic Control, vol. 54, no. 8, pp. 1951-1955, 2009. 